\title{
Analyzing Apomorphine-Mediated Effects in a Cell Model for Parkinson's Disease with Partial Least Squares Structure Equation Modeling
}

\author{
DANIELE PEPE, and JIN HWAN DO ${ }^{2}$
}

\begin{abstract}
Genome-wide gene expression data for cell model of Parkinson's disease (PD) have considerably improved our understanding of the underlying molecular mechanisms involved in cell death during PD neurodegeneration. Apomorphine (APOM), a nonselective dopamine agonist, has been used to treat patients with advanced PD showing no response to levodopa or other dopamine agonists. Although APOM plays a role as a free radical scavenger with neuroprotective effect, it has been reported that long-term use of APOM in PD treatment brings about side effects such as nausea and orthostatic hypotension. For safe use of APOM in PD treatment, it is crucial to understand the underlying molecular mechanisms of APOM in PD. In this study, two groups of microarray data including PD cell model and APOM added PD cell model were used to survey mediation effects of APOM in PD cell model. Mediation analysis between disease genes obtained from PD cell model and drug genes obtained from APOM added PD cell model was carried out with shortest path model on KEGG (Kyoto Encyclopedia of Genes and Genomes) pathways and partial least squares structure equation modeling. Our results suggest that drug genes responding to APOM might contribute to negative regulation of disease genes by direct or indirect ways.
\end{abstract}

Keywords: apomorphine meditation, Parkinson's disease, partial least squares, structural equation modeling.

\section{INTRODUCTION}

$\mathbf{P}$ ARKINSON'S DISEASE (PD), the second most prevalent neurodegenerative disease worldwide, is characterized by motor symptoms such as tremor at rest, bradykinesia, rigidity, and postural instability (Sui et al., 2019). Previous studies have demonstrated extensive loss of dopaminergic neurons in the substantia nigra when PD is diagnosed clinically after developing motor dysfunction (Tolosa et al., 2009). Therefore, increase of dopamine in the brain has been used as a basic strategy for treatment of initial PD. Supply of dopamine to the brain can be carried out by levodopa (L-dihydroxyphenylalanine), which can cross the blood-brain barrier as a natural dopamine precursor. However, long-term administration of levodopa may result in drug resistance and aggravation of symptoms. Thus, advanced PD patients with persistent and disabling motor fluctuations have

\footnotetext{
${ }^{1}$ Department of Oncology, KU Leuven, Leuven, Belgium.

${ }^{2}$ Department of Biomolecular and Chemical Engineering, DongYang University, Yeongju, Korea.
} 
been treated with apomorphine (APOM) instead of levodopa (De Gaspari et al., 2006; Antonini et al., 2011; Carron et al., 2011). APOM is not only a nonselective dopamine agonist that stimulates $D_{1^{-}}$-like $\left(D_{1}, D_{5}\right)$ and $D_{2^{-}}$ like $\left(\mathrm{D}_{2}, \mathrm{D}_{3}, \mathrm{D}_{4}\right)$ receptors, but also a free radical scavenger (Ribarič, 2012). It has been proposed that the dyskinetic effect of APOM on PD patients might be mediated by excessive activation of afferents to centromedian-striatopallidal or pallidal-pedunculopontine pathways (Merello et al., 1999).

Despite the beneficial effect of APOM on patients with advanced PD, its molecular mechanism at cellular level remains unclear. In addition, prolonged use of APOM might result in side effects such as orthostatic hypotension, nausea, and fibrotic nodules at the site of APOM injection (Deleu et al., 2004). For safe use of APOM in PD patients, it is necessary to understand the underlying molecular mechanisms of APOM in PD. Genome-wide gene expressions in PD cell model and APOM-treated PD cell model have been investigated with a commercial whole-genome microarray (human HT-12 expression v.4 bead array; Illumina, Inc.) in our previous studies (Kim et al., 2013; Do, 2017). The PD cell model used in those studies was human neuroblastoma SH-SY5Y cells treated with 1-methyl-4-phenyl-pyridium (MPP ${ }^{+}$) that could mimic many aspects of dopaminergic (DAergic) neuronal death observed in PD (Choi et al., 2014; Do, 2014). The aim of this study was to determine the molecular function of APOM in PD cell model by analyzing interactions of disease genes related to PD and drug (APOM) genes through the shortest path model on KEGG (Kyoto Encyclopedia of Genes and Genomes) pathways and partial least squares structure equation modeling (PLS-SEM) (Pepe and Burzykowski, 2017). For this, two groups of time-series microarray data, that is, $\mathrm{MPP}^{+}$treatment group and $\mathrm{MPP}^{+}+\mathrm{APOM}$ treatment group, were used from our previous studies (Kim et al., 2013; Do, 2017).

As MPP ${ }^{+}$-treated SH-SY5Y cells have been extensively used as PD cell model, their gene expression profiles can be used for detecting PD-associated genes. In a similar way, gene expression profiles of $\mathrm{MPP}^{+}$treated SH-SY5Y cells with APOM were able to discover genes associated with function of the drug (APOM). Expression values for these two groups of genes, that is, disease-associated genes (disease genes) and drug-associated genes (drug genes), were utilized to explore the role of APOM in PD cell model. Number of disease genes and drug genes were 808 and 935, respectively. The number of common genes shared by the two groups was 175 . Among these 175 common genes, expression profiles of 47 genes were directly affected by APOM. The analysis of shortest paths between disease-specific and drug (APOM)specific genes resulted in the identification of 948 mediator genes linking them. These mediator genes might be associated with the underlying molecular mechanism or cellular process of the relationship between disease-specific and drug (APOM)-specific genes. Indirect or mediated effects of mediator genes were tested by PLS-SEM known to be suitable for the analysis of mediating variables.

Results of PLS-SEM mediation analysis suggested that if global expression level of drug genes increased, global expression level of disease genes decreased. Direct effect, indirect effect, and total effect of drug genes on disease genes were $-0.26,-0.35$, and -0.61 , respectively. The indirect effect was carried out by mediator genes, many of them were involved in pathways including ras/dopaminergic synapse/oxytocin/ insulin/apelin/sphingolipid signaling pathways. This indicates that drug-specific genes might regulate disease-specific genes through various signaling pathways. Our result might contribute to the understanding of molecular mechanisms of APOM in PD patients.

\section{METHODS}

\subsection{Identification of disease and drug genes from time course microarray data}

Two groups of time-series microarray data (i.e., gene expression data for $\mathrm{MPP}^{+}$-treated SH-SY5Y cells without APOM and with APOM) were used from the study of Kim et al. (2013) and Do (2017), respectively. To find genes showing significant temporal expression changes in the time-series microarray data, Bioconductor package maSigPro (Conesa et al., 2006) was used. This program identifies differentially expressed genes from time-series microarray data through a two-step regression approach including adjustment of a global model and selection of genes showing significantly different profile. Analysis of singleand multi-series time course microarray data is available in maSigPro. The analysis of single-series time course was carried out to find genes with significant temporal expression changes for $\mathrm{MPP}^{+}$-treated $\mathrm{SH}$ SY5Y cells without APOM and with APOM, respectively. Analysis of multi-series time course was also carried out to identify genes with significantly different profiles between MPP $^{+}$-treated SH-SY5Y cells without APOM and with APOM. 


\subsection{Shortest path analysis between drug-specific and disease-specific genes}

Genes showing significant temporal expression in MPP ${ }^{+}$-treated SH-SY5Y cells can be considered as disease genes because these cells have been utilized as a cell model for PD (Do, 2016). On the contrary, genes with significant temporal expression in $\mathrm{MPP}^{+}$-treated SH-SY5Y cells with APOM can be regarded as drug genes because APOM has a neuroprotective effect on patients with advanced PD (De Gaspari et al., 2006; Antonini et al., 2011; Carron et al., 2011). Common genes between disease genes and drug genes can be considered as disease genes on which APOM act directly or as disease genes on which the drug does not have any effect. Drug-specific and disease-specific genes represent disease genes and drug genes except for those common genes, respectively. Indirect association between drug-specific and disease-specific genes could be understood by inspecting for connections between these two groups of genes. The assumption behind the search is that drug-specific genes can act on disease genes. The reason is that treatment with a drug, that is, APOM, should have an effect on disease genes directly or indirectly by other genes.

All KEGG pathways were joined in a unique graph using R packages graphite and KEGGgraph. Direct shortest paths on the KEGG graph between each any drug-specific genes and disease-specific genes were discovered by using $\mathrm{R}$ package igraph. To evaluate if the found shortest paths could change significantly with time for any gene that receives a connection in the shortest path, two different equations were generated as illustrated hereunder:

$$
\begin{aligned}
& \text { Model 1: } \\
& \mathrm{G}_{\mathrm{i}}=\beta_{\text {in }} \mathrm{G}_{\mathrm{n}}+\mathrm{E}_{\mathrm{i}} \\
& \text { Model } 2: \\
& \mathrm{G}_{\mathrm{i}}=\beta_{\text {in }} \mathrm{G}_{\mathrm{n}}+\gamma_{\mathrm{i}} \mathrm{T}+\mathrm{E}_{\mathrm{i}}
\end{aligned}
$$

where $\mathrm{G}_{\mathrm{i}}$ is the $i$ th gene influenced by the $n$th gene $\mathrm{G}_{\mathrm{n}} ; \beta_{\text {in }}$ is the path coefficient between $\mathrm{G}_{\mathrm{i}}$ and $\mathrm{G}_{\mathrm{n}}$; $\mathrm{T}$ is the time and $\gamma_{i}$ the path coefficient between $G_{i}$ and $T ; E_{i}$ is the term that encloses the unexplained part in the model of $G_{i}$. The difference in the representation of the gene $G_{i}$ between the two equations is in the consideration of time $\mathrm{T}$ in one model. Every equation of the shortest path constitutes the shortest path model. All models generated for a specific shortest path are fitted by SEM as in our previous studies (Pepe and Grassi, 2014; Pepe and Do, 2015, 2016).

To evaluate if time had an effect on the shortest path, a hypothesis test was performed to compare modelimplied covariance matrices of the model with time and without the time. The statistical significance was measured using likelihood ratio test. If value of $p$ is $<0.05$ after Benjamin-Hochberg correction for the number of tested shortest paths, the shortest path is considered to change significantly with time. Only significant shortest paths are kept.

\subsection{Mediation analysis with PLS-SEM}

Mediation analysis with PLS-SEM was performed to understand if the drug (APOM) had an effect on the disease state cells, that is, MPP ${ }^{+}$-treated SH-SY5Y cells. To this end, three latent variables (i.e., "Treat," "Dis," and "Med") were built. Indicator genes for each latent variable were obtained from the united graph of total significant shortest paths on the KEGG pathway. Three groups of genes (i.e., 169 drug genes, 110 disease genes, and 948 mediator genes) found in shortest path analysis between drug-specific genes and disease-specific genes were used as indicator genes for each latent variable. That is, indicators of "Treat," "Dis," and "Med" were 169 drug genes, 110 disease genes, and 948 mediator genes, respectively. Not all genes in the set were used as indicator genes because the number of samples in the dataset was less than the number of genes in each set. For selecting indicator genes, a hierarchical clustering analysis based on correlation was performed.

After defining the latent variable, a mediation model was generated in which "Treat" was the independent variable and "Dis" was the dependent variable. The analysis allowed us to determine if the treatment had an effect on the disease and how it was determined if directly, by the mediator in both ways. Using PLS-SEM and the set of genes belonging to each latent variable, a mediation model was created and evaluated. These genes were considered as reflective indicators. This means that indicators are caused by latent variable. PLS models are characterized by two models: structural model and measurement model. The first one analyzes relationships between latent variables, whereas the second examines one relationships 
between observed (indicator) variables and latent variables. The structural model can be mathematically represented as follows:

$$
\mathrm{LV}=\mathrm{LV} \times \mathrm{B}+\mathrm{E}
$$

where LV is the matrix representing the latent variables, B is the matrix of coefficients between latent variables, and $\mathrm{E}$ is the matrix of residuals. The measurement model for a particular latent variable $\mathrm{n}$ can be represented as follows:

$$
\mathrm{OV}_{\mathrm{n}}=\mathrm{LV}_{\mathrm{n}} \mathrm{w}_{\mathrm{n}}^{\mathrm{T}}+\mathrm{E}_{\mathrm{n}}
$$

where $\mathrm{OV}_{\mathrm{n}}$ is the block matrix of observed variables that generates latent variable $\mathrm{LV}_{\mathrm{n}} ; \mathrm{w}_{\mathrm{n}}^{\mathrm{T}}$ is the matrix of correlation coefficients between $\mathrm{OV}_{\mathrm{n}}$ and $\mathrm{LV}_{\mathrm{n}}$ computed by least squares; and $\mathrm{E}_{\mathrm{n}}$ is the error term. In the procedure we used, latent variables were normal standard variables. Statistical significance of path coefficients was determined by bootstrap (Monecke and Leisch, 2012). The path coefficient represents estimated change in the dependent latent variable for a unit change of the independent latent variable.

For model evaluation, two types of measures exist. The first ones are for evaluating the structure model [Eq. (1)]. The model was constituted by connections between latent variables. The second ones are for evaluating the goodness of the measurement model [Eq. (2)]. The model was constituted only by latent variables and their relationships with indicators. For structure model evaluation, $R^{2}$ was used. It indicates how much variance of the endogenous latent variable is explained by independent latent variables. It is possible to classify the goodness of model based on the following criteria according to Sanchez (2013): (1) Low: $R^{2}<0.30$ (for some authors $<0.20$ ); (2) Moderate: $0.30<R^{2}<0.60$ (or $0.20<R^{2}<0.50$ ); and (3) High: $R^{2}>0.60$ (or $>0.5$ ). For measurement models, Dillon-Goldstein's rho and communality were used. The Dillon-Goldstein's rho explains to which extent the constructs can explain indicators. Its acceptable value should be $>0.7$. The communality corresponds to squared correlations between latent variables and its indicators. In other words, it measures shared variance between latent variables and their indicators. An acceptable value of communality is 0.5 .

\section{RESULTS}

\subsection{Exploring disease and drug genes from microarray data}

As SH-SY5Y cells exposed to $\mathrm{MPP}^{+}$mimic PD, responsive genes to $\mathrm{MPP}^{+}$treatment may be considered as disease-associated genes or simply disease genes. On the contrary, responsive genes to treatment of $\mathrm{MPP}^{+}$with APOM might be regarded as drug-associated genes or simply drug genes because APOM is a nonselective dopamine agonist for PD. Therefore, disease or drug genes could be detected by microarray experiment that allows us to measure gene expression response or change at genomic scale. In this study, two time-series microarray data were used from our previous studies (Kim et al., 2013; Do, 2017) for the detection of disease genes and drug (APOM) genes in PD cell model. Both microarray data consisted of expression values measured at six time points $\left(0,3,6,9,12\right.$, and 24 hours) from $\mathrm{MPP}^{+}$-treated SH-SY5Y cells and $\mathrm{MPP}^{+}$-treated SH-SY5Y cells with APOM for a total of 30,486 genes in the microarray. Genes with differential expression profile were identified with a Bioconductor package maSigPro (Conesa et al., 2006) known to support the analysis of time-series microarray data.

Disease genes were detected from the time-series microarray experiment data of $\mathrm{MPP}^{+}$-treated SH-SY5Y cells. The number of disease genes was $808, \sim 2.7 \%$ of total genes on the microarray. Drug (APOM) genes were also detected from the time-series microarray experiment data of SH-SY5Y cells exposed to MPP ${ }^{+}$ with APOM. The number of drug genes was $935, \sim 3.1 \%$ of total genes on the microarray. There were 175 common genes shared by disease genes and drug genes (Fig. 1), $\sim 11.2 \%$ of total response genes (disease genes+drug genes). These common genes can be interpreted as disease genes on which the drug (APOM) act directly or as disease genes on which the drug (APOM) does not have any effect.

To understand on which genes the drug (APOM) could act directly, a multi-series analysis was performed for the two time-series microarray data. Fifty-five genes showed differential profiles between the 2 groups of microarray data, of which 47 belong to the 175 common genes. Therefore, these 47 genes can be considered as disease genes on which the drug (APOM) can act directly. They are given in Table 1. Gene Ontology (GO) terms that were significantly overrepresented in these 47 genes were estimated with a webbased program Gorilla (Eden et al., 2009). Results are given in Table 2. GO terms for biological process, 


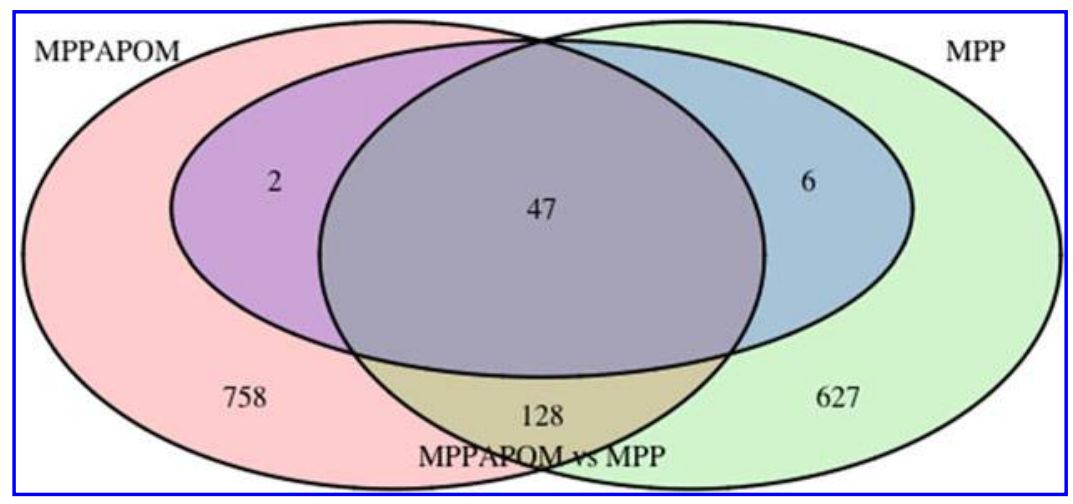

FIG. 1. Venn diagram showing drug genes (MPPAPOM) and disease genes (MPP) detected from two groups of timeseries microarray data.

immune response-regulating cell surface receptor signaling pathway involved in phagocytosis (GO:0002433), positive regulation of peptidase activity (GO:0010952), and positive regulation of proteolysis (GO:0045826) showed significant enrichment scores with $p$-value $<1.0 \mathrm{E}-3$. Enriched GO terms for cellular component included external organelle (GO:0043230), membrane-bounded organelle (GO:0043227), and azurophil granule lumen (GO:0035578). This suggested that APOM in PD might directly target genes associated with autophagic and phagocytic pathways.

\subsection{Shortest path analysis between drug-specific and disease-specific genes}

Among a total of 1568 response genes including disease and drug genes, there were only 175 common genes (Fig. 1). That is, the number of disease-specific genes was 633, whereas 760 genes were specific to the drug (APOM). Drug-specific genes might be indirectly associated with disease-specific genes. Indirect association between drug-specific and disease-specific genes could be understood by looking for connections between the two groups of genes. Using KEGG network obtained by merging all KEGG pathways (with R package graphite and KEGGgraph) and the shortest paths between drug-specific genes and disease-specific genes, it was possible to discover how the drug (APOM) effect propagated until disease genes were reached. The KEGG network included 5238 nodes and 73,070 connections. However, not all genes in the graph were present in the microarray experiment. For this reason, 77 genes were excluded from the graph. Therefore, analysis of shortest path model was performed on the KEGG graph constituted by 5161 genes and 71,636 connections. The number of direct shortest paths between drug-specific and disease-specific genes was 18,704.

To evaluate if the found shortest paths changed significantly with time for any gene that received a connection in the shortest path, an SEM analysis on each shortest path was performed (see Section 2.2 for details). The total number of significant shortest paths was 5263. Their union generated a graph of 1227 genes and 4602 connections. These 1227 genes of the graph can be distinguished into three groups of genes: (1) 169 drug genes from which the effect of drug starts, (2) 110 disease genes, and (3) 948 mediator genes that connect the drug and disease genes. To explore enriched pathways in each group of genes, $\mathrm{R}$ package clusterProfiler (Yu et al., 2012) was used. Figures 2 and 3 show significantly enriched KEGG pathways in 169 drug genes and 948 mediator genes, respectively. No KEGG pathway was enriched for 110 disease genes. Enriched pathways for 169 drug genes included carbon/amino sugar and nucleotide sugar/inositol phosphate metabolism, protein processing in endoplasmic reticulum, estrogen/cAMP/HIF-1/ glucagon signaling pathways, and so on. In the case of 948 mediator genes, there are many enriched signaling pathways, including ras/dopaminergic synapse/oxytocin/insulin/apelin/sphingolipid signaling pathways. This indicates that drug genes might regulate disease genes through mediator genes that are related to many signaling pathways.

\subsection{Mediation analysis of mediator genes with PLS-SEM}

In the previous section, three groups of genes were found through shortest path analysis between drugspecific genes and disease-specific genes (i.e., 169 drug genes, 110 disease genes, and 948 mediator genes). By using these three groups of genes, mediation analysis with PLS-SEM was performed to understand if 
Table 1. Forty-Seven Disease Genes Directly Affected by Apomorphine

\begin{tabular}{|c|c|}
\hline Gene & Description \\
\hline$M A P K 3$ & Mitogen-activated protein kinase 3 (MAPK3), mRNA \\
\hline$A B C G 1$ & ATP-binding cassette, subfamily $\mathrm{G}$ (WHITE), member 1 (ABCG1), transcript variant 2 , mRNA \\
\hline ACO1 & Aconitase 1 , soluble (ACO1), mRNA \\
\hline ANKRD6 & Ankyrin repeat domain 6 (ANKRD6), mRNA \\
\hline C18orf55 & chromosome 18 open reading frame 55 (C18orf55), mRNA \\
\hline$C C D C 24$ & Coiled-coil domain containing 24 (CCDC24), mRNA. \\
\hline СМТМ & CKLF-like MARVEL transmembrane domain containing 8 (CMTM8), mRNA \\
\hline CORO2A & Coronin, actin-binding protein, $2 \mathrm{~A}(\mathrm{CORO} 2 \mathrm{~A})$, transcript variant 1, mRNA \\
\hline CTSH & Cathepsin H (CTSH), transcript variant 1 , mRNA \\
\hline CXXC6 & CXXC finger 6 (CXXC6), mRNA \\
\hline CYCS & Cytochrome c, somatic (CYCS), nuclear gene encoding mitochondrial protein, mRNA \\
\hline CYFIP2 & Cytoplasmic FMR1 interacting protein 2 (CYFIP2), transcript variant 1, mRNA \\
\hline EPHX2 & Epoxide hydrolase 2, cytoplasmic (EPHX2), mRNA \\
\hline$F A B P 5$ & Fatty acid-binding protein 5 (psoriasis-associated) (FABP5), mRNA \\
\hline FAM136A & Family with sequence similarity 136, member A (FAM136A), mRNA \\
\hline GALK1 & Galactokinase 1 (GALK1), mRNA \\
\hline$H 2 A F J$ & $\mathrm{H} 2 \mathrm{~A}$ histone family, member $\mathrm{J}$ (H2AFJ), transcript variant 1 , mRNA \\
\hline$H E B P 1$ & Heme-binding protein 1 (HEBP1), mRNA \\
\hline HIST1H2BK & Histone cluster 1, H2bk (HIST1H2BK), mRNA \\
\hline HIST2H2AA4 & Histone cluster 2, H2aa4 (HIST2H2AA4), mRNA \\
\hline HOXB6 & Homeo box B6 (HOXB6), transcript variant 3, mRNA \\
\hline INSMI & Insulinoma-associated 1 (INSM1), mRNA \\
\hline KLF15 & Kruppel-like factor 15 (KLF15), mRNA \\
\hline$L M T K 3$ & PREDICTED: lemur tyrosine kinase 3 (LMTK3), mRNA \\
\hline LOC345041 & PREDICTED: misc_RNA (LOC345041), miscRNA \\
\hline LOC 388588 & PREDICTED: hypothetical LOC388588, transcript variant 2 (LOC388588), mRNA \\
\hline LOC 389523 & PREDICTED: similar to opposite strand transcription unit to Stag3 (LOC389523), mRNA \\
\hline LOC441046 & Glucuronidase, beta pseudogene (LOC441046), noncoding RNA \\
\hline LOC647741 & PREDICTED: hypothetical LOC647741 (LOC647741), mRNA \\
\hline$M A G E C 2$ & Melanoma antigen family C, 2 (MAGEC2), mRNA \\
\hline МСМЗАР & Minichromosome maintenance complex component 3 associated protein (MCM3AP), mRNA \\
\hline MIR1974 & MicroRNA 1974 (MIR1974), microRNA \\
\hline MTSS1 & Metastasis suppressor 1 (MTSS1), mRNA. \\
\hline NDUFV1 & NADH dehydrogenase (ubiquinone) flavoprotein 1, $51 \mathrm{kDa}$ (NDUFV1), mRNA \\
\hline PACSIN3 & Protein kinase $\mathrm{C}$ and casein kinase substrate in neurons 3 (PACSIN3), mRNA \\
\hline PAOX & Polyamine oxidase (exo-N4-amino) (PAOX), transcript variant 5, mRNA \\
\hline POTEF & POTE ankyrin domain family, member F (POTEF), mRNA \\
\hline$P R K C D$ & Protein kinase $\mathrm{C}$, delta (PRKCD), transcript variant 1, mRNA \\
\hline RGS10 & Regulator of G-protein signaling 10 (RGS10), transcript variant 1, mRNA \\
\hline RIMS3 & Regulating synaptic membrane exocytosis 3 (RIMS3), mRNA \\
\hline RINL & Ras and Rab interactor-like (RINL), mRNA \\
\hline RNASET2 & Ribonuclease T2 (RNASET2), mRNA \\
\hline$R O G D I$ & Rogdi homolog (Drosophila) (ROGDI), mRNA \\
\hline SCRG1 & Scrapie responsive protein 1 (SCRG1), mRNA \\
\hline SFRS3 & Splicing factor, arginine/serine-rich 3 (SFRS3), mRNA \\
\hline THBS3 & Thrombospondin 3 (THBS3), mRNA \\
\hline TLE6 & Transducin-like enhancer of split 6 [E(sp1) homolog, Drosophila] (TLE6), mRNA \\
\hline
\end{tabular}

drug genes have an effect on disease genes. To this end, 3 latent variables of "Treat," "Dis," and "Med" were built with 169 drug genes, 110 disease genes, and 948 mediator genes as their reflective indicators, respectively. Not all genes in the set were used because the number of samples in the dataset was less than the number of genes in each set. For selecting genes, a hierarchical clustering analysis based on correlation was performed. In the next step, PLS-SEM model was created and fitted. PLS-SEM is characterized by two models: structural model and measurement model. The first one analyzes relationships between latent 
Table 2. Enriched Gene Ontology Terms Discovered by Gorilla Program in 47 Disease Genes Directly Affected by the Drug (APOMORPHine) Versus Background Gene Set, Including All Genes on the Array

\begin{tabular}{lllr}
\hline GO & GO term & \multicolumn{1}{c}{ Description } & $\mathrm{p}$ \\
\hline BP & GO:0002433 & Immune response-regulating cell surface receptor signaling pathway & $4.75 \mathrm{E}-04$ \\
& & involved in phagocytosis \\
& GO:0038096 & Fc-gamma receptor signaling pathway involved in phagocytosis & $4.75 \mathrm{E}-04$ \\
& GO:0002431 & Fc receptor mediated stimulatory signaling pathway & $5.16 \mathrm{E}-04$ \\
GO:0038094 & Fc-gamma receptor signaling pathway & $5.37 \mathrm{E}-04$ \\
& GO:0035850 & Epithelial cell differentiation involved in kidney development & $6.17 \mathrm{E}-04$ \\
GO:0010952 & Positive regulation of peptidase activity & $8.24 \mathrm{E}-04$ \\
GO:0045862 & Positive regulation of proteolysis & $9.98 \mathrm{E}-04$ \\
CC & GO:0031982 & Vesicle & $4.22 \mathrm{E}-05$ \\
& GO:0070062 & Extracellular exosome & $3.48 \mathrm{E}-04$ \\
& GO:1903561 & Extracellular vesicle & $3.87 \mathrm{E}-04$ \\
& GO:0043230 & Extracellular organelle & $3.89 \mathrm{E}-04$ \\
& GO:0043227 & Membrane-bounded organelle & $4.99 \mathrm{E}-04$ \\
& GO:0044421 & Extracellular region part & $5.56 \mathrm{E}-04$ \\
& GO:0035578 & Azurophil granule lumen & $9.90 \mathrm{E}-04$ \\
\hline
\end{tabular}

BP, biological process; CC, cellular component; GO, Gene Ontology.

variables, whereas the second one examines relationships between indicator variables (here, genes) and the latent variables. Therefore, feasibility measure of PLS-SEM is separately evaluated for structural model and measurement model.

The feasibility of measurement model was evaluated by Dillon-Goldstein rho value to explain to which extent latent variables could explain indicator variables. An acceptable value should be $>0.7$. In our case, Dillon-Goldstein rho values for the measurement model were 0.98, 0.95, and 0.99 for latent variables of "Treat," "Dis," and "Med," respectively. Another important feasibility measure is communality, which corresponds to squared correlations between latent variables and its indicators. In other words, it measured shared variance between latent variables and their indicators. An acceptable value of communality is 0.5 . In our case, communality values were $0.85,0.90$, and 0.85 for latent variables of "Treat," "Dis," and "Med," respectively. For structure model evaluation, we used $R^{2}$ to show how much variance of the endogenous latent variable was explained by independent latent variables. In our model, we had only an independent latent variable, "Treat." Mean $R^{2}$ was 0.52 , a moderate value of $R^{2}$.

When analyzing the model, it is important to consider estimated value of path coefficient that represents the response of dependent variable to a unit change in an independent variable when other variables in the

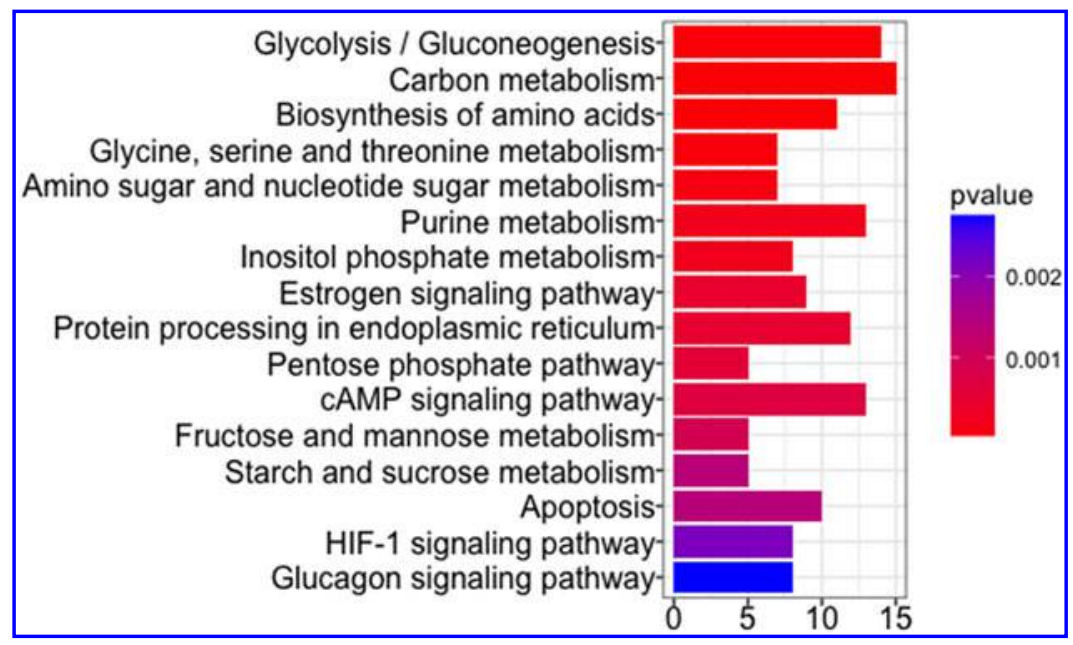

FIG. 2. KEGG pathway enrichment analysis for 169 drug genes on the united graph of significant shortest paths. KEGG, Kyoto Encyclopedia of Genes and Genomes. 


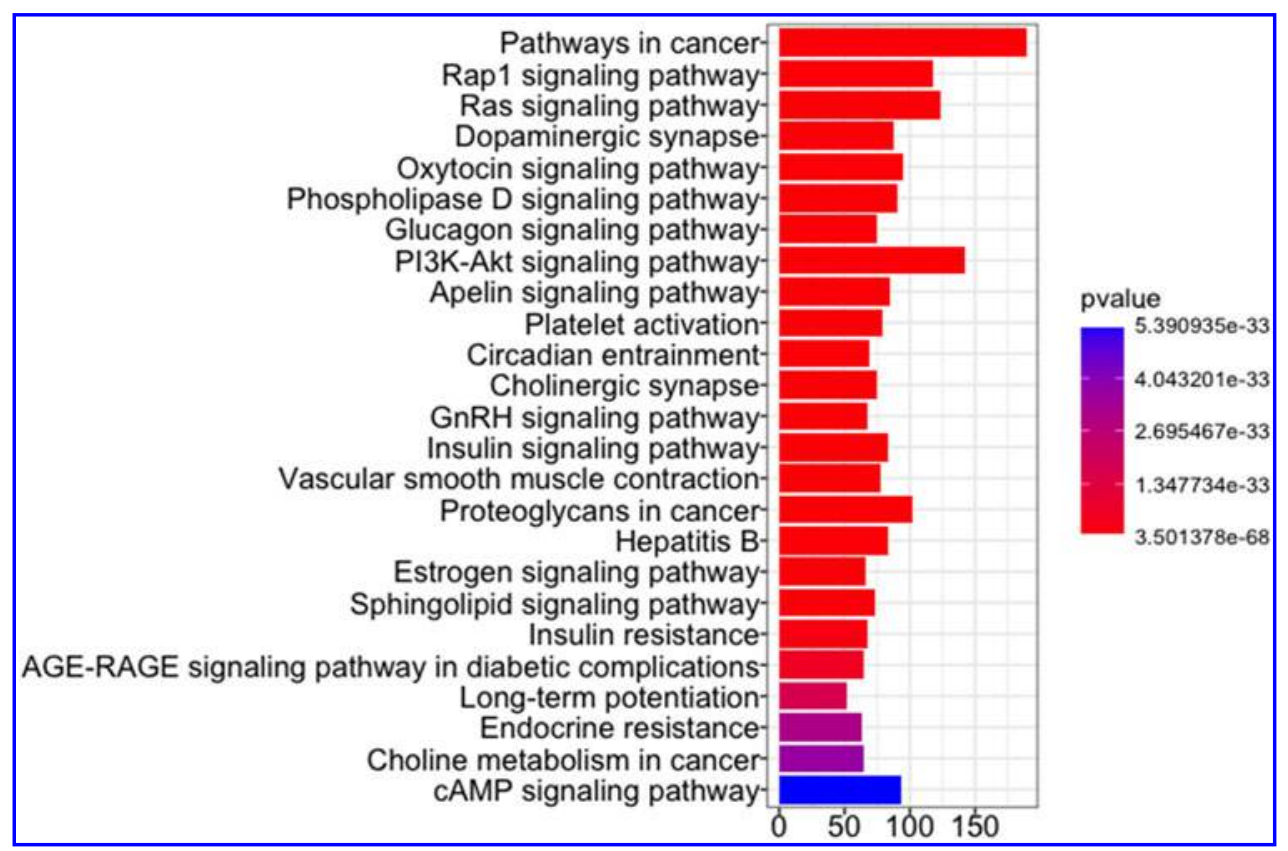

FIG. 3. KEGG enrichment analysis for 948 mediator genes on the united graph of significant shortest paths.

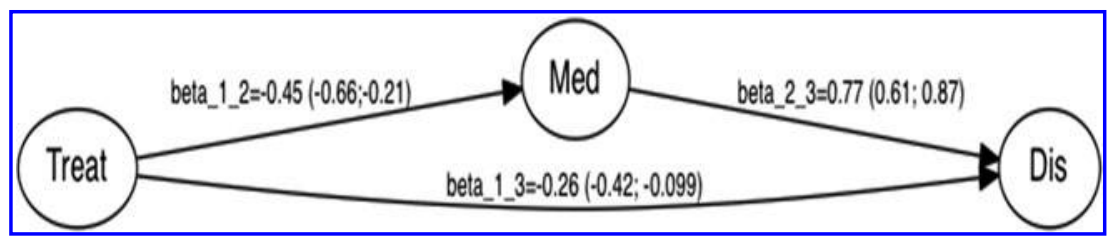

FIG. 4. Path diagram for structure model showing relationships among three latent variables. The number on arrow represents path coefficient with $95 \%$ confidence interval shown inside parenthesis.

model are fixed. The path coefficient can be interpreted as the connection strength or effect strength between latent variables. The significance of path coefficient was evaluated with a bootstrap approach. As given in Figure 4, all path coefficients are significant if 95\% confidence intervals do not include zero. The direct effect from latent variable "Treat" to "Dis" was -0.26 . The indirect effect through "Med" was -0.35 (by multiplying path coefficients involved in the indirect path, i.e., $-0.45 \times 0.77$ ). Total effect (direct effect + indirect effect) was -0.61 . This means that, if we increase global expression of drug genes, they can reduce global expression of disease genes by direct and indirect ways. This indicates that drug genes responding to APOM might contribute to negative regulation of disease genes by direct or indirect ways.

\section{DISCUSSION AND CONCLUSIONS}

Unlike levodopa that requires conversion into dopamine to be effective for PD, APOM directly plays a role as a nonselective dopamine agonist. APOM is used mainly for advanced PD patients showing no response to levodopa or other agonists (Okun and Foote, 2010). APOM has functions as a potent antioxidant and a free radical scavenger besides its receptor-mediated action (Gassen et al., 1996). However, it has been reported that long-term use of APOM in PD treatment brings about side effects such as nausea, orthostatic hypotension, and fibrotic nodules at the site of APOM injection (Deleu et al., 2004). For safe use of APOM in PD treatment, it is essential to understand the molecular mechanisms involved in its effect.

In this study, we examined mediated effects of APOM in PD cell model by using two groups of timeseries microarray data. Disease genes and drug genes were identified from microarray data of $\mathrm{MPP}^{+}$-treated SH-SY5Y cells and APOM-added MPP ${ }^{+}$-treated SH-SY5Y cells, respectively. Numbers of disease genes 
and drug genes were 808 and 935 , respectively. The number of common genes between disease and drug genes was $175, \sim 11.2 \%$ of total response genes (disease genes and drug genes). Among these 175 common genes, expression profiles of 47 genes showed significant changes by the drug (APOM). Therefore, these 47 genes could be considered as disease genes on which APOM can act directly. Enriched GO terms in these 47 genes were GO:0002433 (immune response-regulating cell surface receptor signaling pathway involved in phagocytosis), GO:0002431 (Fc receptor-mediated stimulatory signaling pathway), GO:0045862 (positive regulation of proteolysis), and so on (Table 2).

Drug-specific and disease-specific genes were obtained after excluding the 175 common genes in their gene group, respectively. Drug-specific and disease-specific genes might be linked by mediator genes. On the graph obtained by merging all KEGG pathways, shortest paths linking drug-specific genes and diseasespecific genes were evaluated with SEM. The number of genes on the graph generated by merging all significant shorted paths was 1,227. These 1,227 genes can be distinguished into 3 groups: 169 drug genes, 110 disease genes, and 948 mediator genes. In the pathway enrichment analysis for 948 mediator genes, many signaling pathways including ras/dopaminergic synapse/oxytocin/insulin/apelin/sphingolipid signaling pathways showed enrichment. This suggests that drug genes might regulate disease genes through mediator genes that are related to signaling pathways.

To explore the role of mediator genes toward disease genes, mediation analysis with PLS-SEM was performed by using expression values of three group of genes (i.e., 169 drug genes, 110 disease genes, and 948 mediator genes). Three latent variables of "Treat," "Dis," and "Med" were built with 169 drug genes, 110 disease genes, and 948 mediator genes as their reflective indicators, respectively. The direct effect from latent variable "Treat" to "Dis" was -0.26 . The indirect effect through "Med" was -0.35 and the total effect was -0.61 (Fig. 4). This indicates that drug genes responding to APOM might contribute to negative regulation of disease genes by direct or indirect ways. Our result supports that APOM has effectiveness as drug on PD cell model in terms of gene expression regulation.

\section{AUTHOR DISCLOSURE STATEMENT}

The authors declare they have no competing financial interests.

\section{FUNDING INFORMATION}

This study was supported by a grant (No. 2012R1A1A2005622) of the Basic Science Research Program through the National Research Foundation (NRF) of Korea funded by the Ministry of Education, Science, and Technology (MEST).

\section{REFERENCES}

Antonini, A., Isaias, I.U., Rodolfi, G., et al. 2011. A 5-year prospective assessment of advanced Parkinson disease patients treated with subcutaneous apomorphine infusion or deep brain stimulation. J. Neurol. 258, 579-585.

Carron, R., Fraix, V., Maineri, C., et al. 2011. High frequency deep brain stimulation of the subthalamic nucleus versus continuous subcutaneous apomorphine infusion therapy: A review. J. Neural Transm. 118, 915-924.

Choi, D.-K., Kim, I.S., and Do, J.H. 2014. Signaling pathway analysis of MPP+-treated human neuroblastoma SHSY5Y cells. Biotechnol. Bioproc. Eng. 19, 332-340.

Conesa, A., Nueda, M.N., Ferrer, A., et al. 2006. maSigPro: A method to identify significantly differential expression profiles in time-course microarray experiments. Bioinformatics 22, 1096-1102.

De Gaspari, D., Siri, C., Landi, A. et al. 2006. Clinical and neuropsychological follow up at 12 months in patients with complicated Parkinson's disease treated with subcutaneous apomorphine infusion or deep brain stimulation of the subthalamic nucleus. J. Neurol. Neurosurg. Psychiatry 77, 450-453.

Deleu, D., Hanssens, Y., and Northway, M.G. 2004. Subcutaneous apomorphine: An evidence-based review of its use in Parkinson's disease. Drugs Aging 21, 687-709.

Do, J. H. 2014. Neurotoxin-induced pathway perturbation in human neuroblastoma SH-EP cells. Mol. Cells $37,672-$ 684.

Do, J.H. 2016. Genome-wide transcriptional response of $\mathrm{MPP}^{+}$-treated human neuroblastoma SH-SY5Y cells to apomorphine. Anim. Cells Syst. 20, 140-151. 
Do, J.H. 2017. Apomorphine-induced pathway perturbation in MPP ${ }^{+}$-treated SHSY cells. AIMS Mol. Sci. 4, $271-287$. Eden, E., Navon, R., Steinfeld, I., et al. 2009. Gorilla: A tool for discovery and visualization of enriched GO terms in ranked gene list. BMC Bioinformatics 10, 48.

Gassen, M. Glinka, Y., Pinchasi, B., et al. 1996. Apomorphine is a highly potent free radical scavenger in rat brain mitochondrial fraction. Eur. J. Pharmacol. 308, 219-225.

Kim, I.S., Choi, D.-K., and Do, J.H. 2013. Genome-wide temporal responses of human neuroblastoma SH-SY5Y cells to $\mathrm{MPP}^{+}$neurotoxity. BioChip J. 7, 247-257.

Merello, M., Lees, A. J., Balej, J., et al. 1999. GPi firing rate modification during beginning-of-dose motor deterioration following acute administration of apomorphine. Mov. Disord. 14, 481-483.

Monecke, A., and Leisch, F. 2012. semPLS: Structural equation modeling using partial least squares. J. Stat. Softw. 48, $1-32$.

Okun, M.S., and Foote, K.D. 2010. Parkinson's disease DBS: What, when, who and why? The time has come to tailor DBS targets. Expert. Rev. Neurother. 10, 1847-1857.

Pepe, D., and Burzykowski, T. 2017. PLS-SEM mediation analysis of gene-expression data for the evaluation of a drug effect, 59-69. In Bracciali, A., Caravagna, G., Gilbert, D. et al., eds. Computational Intelligence Methods for Bioinformatics and Biostatistics. Springer, Cham.

Pepe, D., and Do, J.H. 2015. Estimation of dysregulated pathway regions in $\mathrm{MPP}^{+}$-treated human neuroblastoma SH-EP cells with structural equation model. BioChip J. 9, 131-138.

Pepe, D., and Do, J.H. 2016. Comparison of perturbed pathways in two different cell models for Parkinson's disease with structural equation model. J. Comput. Biol. 23, 90-101.

Pepe, D., and Grassi M. 2014. Investigating perturbed pathway modules from gene expression data via structural equation models. BMC Bioinformatics $15,1-15$.

Ribarič, S. 2012. The pharmacological properties and therapeutic use of apomorphine. Molecules 17, 5289-5309.

Sanchez, G. 2013. PLS Path Modeling with R. Trowchez Editions, Berkeley.

Sui, X., Zhou, C., Li, J., et al. 2019. Hyposmia as a predictive marker of Parkinson's disease: A systematic review and meta-analysis. Biomed. Res. Int. [Epub ahead of print]; DOI: 10.1155/2019/3753786.

Tolosa, E., Gaig, C., Santamaría, J., et al. 2009. Diagnosis and the premotor phase of Parkinson disease. Neurology 72, S12-S20.

Yu, G., Wang, L.G., Han, Y., et al. 2012. clusterProfiler: An R package for comparing biological themes among clusters. $\underline{O M I C S} 16,284-287$.

Address correspondence to:

Prof. Jin Hwan Do

Department of Biomolecular and Chemical Engineering

DongYang University

Yeongju 36040

Korea

E-mail: jinhwando@dyu.ac.kr 